\title{
Epidemiologia das neoplasias intracranianas no Hospital do Servidor Público Estadual de São Paulo: 2010-2012
}

\author{
Daniella Brito Rodrigues', Lorena de Oliveira Lima', \\ Edmundo Luis Rodrigues Pereira², Ulysses de Oliveira Souza ${ }^{3}$, \\ Matheus Fernandes de Oliveira ${ }^{3}$, Alessandra de Moura Lima ${ }^{4}$, José Marcus Rotta ${ }^{5}$ \\ Serviço de Neurocirurgia do Hospital do Servidor Público Estadual de São Paulo, São Paulo, SP, Brasil.
}

\section{RESUMO}

Objetivo: Analisar aspectos clínicos e epidemiológicos dos tumores cerebrais primários e metastáticos entre os pacientes atendidos no Hospital do Servidor Público Estadual de São Paulo, durante os anos de 2010 a 2012. Método: Estudo transversal, retrospectivo, do tipo observacional, com análise de 369 casos de neoplasias intracranianas de acordo com os dados obtidos no setor de registro hospitalar, nos prontuários da instituição e registros anatomopatológicos dos pacientes tratados cirurgicamente. Resultados: Tumores cerebrais primários corresponderam a 69\% da amostra; houve predomínio do sexo feminino; a média de idade foi de 62 anos, com pico na incidência na faixa etária de 41 a 64 anos. Meningiomas foram os tumores cerebrais primários mais encontrados $(23 \%)$, seguidos pelos gliomas (21\%). Cânceres de pulmão, de mama e do trato gastrointestinal foram, em ordem decrescente, as localizações primárias mais frequentes das metástases; estas foram múltiplas em somente $26 \%$ dos casos e tiveram predileção pelo lobo frontal e pelo cerebelo. Conclusão: Tumores intracranianos primários foram predominantes na casuística; desses, os meningiomas e os gliomas corresponderam aos principais tipos histológicos. As neoplasias de pulmão, mama e do trato gastrointestinal foram, em ordem decrescente, as localizações primárias mais frequentes das metástases intracranianas; estas tiveram predileção pelo lobo frontal e cerebelo e foram únicas, em sua maioria.

\section{PALAVRAS-CHAVE}

Neoplasias encefálicas, metástase neoplásica, epidemiologia.

\begin{abstract}
Epidemiology of intracranial neoplasms in Hospital of Servidor Público Estadual of São Paulo: 2010-2012

Objective: To perform an epidemiological analysis about primary and metastatic brain tumors among patients treated at the Hospital of Servidor Público of São Paulo during the years 2010 to 2012. Method: Epidemiological study descriptive, retrospective and observational of medical records of 369 patients who underwent treatment for intracranial tumors primary or metastatic brain tumors between the years 2010 and 2012 at Hospital of Servidor Público of São Paulo (HSPE-SP), according to the data obtained in the field of hospital records, the records of the institution and pathological records of patients treated surgically. Results: Primary brain tumors accounted for $69 \%$ of the sample was predominantly female, the average age was 62 years, with peak incidence in the age group 41-64 years. Meningiomas were found most primary brain tumors (23\%), followed by gliomas (21\%). Lung cancer, breast and gastrointestinal tract were, in descending order, the most frequent primary locations of metastases, these were multiple in only $26 \%$ of cases and had a predilection for frontal lobe and the cerebellum. Conclusion: Primary intracranial tumors were predominant in the sample, meningiomas and gliomas corresponded to the main histological types. Cancers of the lung, breast and gastrointestinal tract were, in descending order, the most frequent primary locations of intracranial metastases; these had predilection for frontal lobe and cerebellum and were, most frequently, single.
\end{abstract}

\section{KEYWORDS}

Brain neoplasms, neoplasm metastasis, epidemiology.

1 Acadêmicas de Medicina da Universidade do Estado do Pará (UEPA), Belém, PA, Brasil.

Neurocirurgião, professor de Neurologia da Universidade Federal do Pará (UFPA), Belém, PA, Brasil.

Médico-residente de Neurocirurgia do Hospital do Servidor Público Estadual de São Paulo (HSPE-SP), São Paulo, SP, Brasil.

Neurocirurgiã do HSPE-SP, São Paulo, SP, Brasil.

5 Diretor do Serviço de Neurocirurgia do HSPE-SP, São Paulo, SP, Brasil. 


\section{Introdução}

Os tumores do sistema nervoso central (SNC) são responsáveis por $1,5 \%$ de todos os cânceres e por 2,4\% de todas as mortes por câncer anualmente. ${ }^{1,2} \mathrm{~A}$ incidência de tumores cerebrais primários é de 11 a 19 para cada 100.000 indivíduos, ${ }^{3}$ enquanto a incidência das metástases cerebrais é estimada em 11:100.000 na população em geral, e estudos em necropsias demonstram que até $25 \%$ dos pacientes portadores de neoplasias apresentam metástases no encéfalo. ${ }^{4} \mathrm{~A}$ incidência e a mortalidade dos tumores do SNC aumentaram na maioria dos países desenvolvidos, principalmente nas faixas etárias mais avançadas, e em grande parte desses países a mortalidade por essas neoplasias ocupa a $12^{\mathrm{a}}$ posição. ${ }^{5}$

Os tumores supratentoriais são os mais frequentes em adultos, e os gliomas (astrocitomas, ependimomas, oligodendrogliomas) são responsáveis por mais de 30\% dos tumores nesse grupo, seguidos pelos meningiomas e schwannomas. Em crianças, a localização mais comum é infratentorial, sendo os meduloblastomas, ependimomas e astrocitomas pilocíticos do cerebelo os tipos histológicos mais frequentes. ${ }^{2}$ A classificação mais utilizada para caracterizar os tumores cerebrais primários é aquela estabelecida pela Organização Mundial da Saúde (OMS); baseada na possível origem celular das neoplasias, subdivide os tumores em quatro graus crescentes de malignidade: grau I a IV. Sendo os tumores graus I e II de baixa gradação, isto é, benignos, enquanto os graus III e IV são considerados malignos. ${ }^{6}$ As neoplasias secundárias são classificadas de acordo com a sua localização primária, e os tumores primários que mais frequentemente evoluem com metástases para o SNC em adultos são o carcinoma broncogênico (principalmente o carcinoma de pequenas células e o adenocarcinoma), o câncer de mama, o carcinoma renal, o melanoma e as neoplasias malignas do trato gastrointestinal, entretanto até $10 \%$ das metástases cerebrais têm origem primária desconhecida. ${ }^{7-10}$ Nos pacientes com idade inferior a 21 anos, as metástases originam-se principalmente dos sarcomas (sarcoma osteogênico, rabdomiossarcoma e sarcoma de Ewing) e dos tumores de células germinativas. ${ }^{4}$

O prognóstico dos tumores cerebrais depende do tipo de tumor, seu grau histológico, localização, idade do paciente e condições fundamentais (status de desempenho de Karnofsky, KPS); nas metástases, acrescentam-se a localização primária, a sensibilidade do tumor à terapia e o número de lesões no SNC como importantes fatores prognósticos., ${ }^{2,11}$

Embora a incidência de neoplasias intracranianas seja inferior a dos demais cânceres, os tumores cerebrais assumem grande importância na oncologia e se destacam no âmbito de novas pesquisas e tratamentos. As metásta- ses intracranianas ainda são consideradas a complicação neurológica mais frequente e temível entre os cânceres. ${ }^{12}$ Além dos elevados índices de déficits funcionais e da importância das metástases cerebrais no prognóstico dos demais cânceres, os tumores cerebrais se destacam no âmbito oncológico devido aos grandes avanços no tratamento nos campos genético e molecular obtidos com as descobertas acerca da etiologia desses tumores. Há um número desproporcional de óbitos e sequelas em comparação com outros tipos de cânceres, o que torna as neoplasias intracranianas um importante problema de saúde pública. Diante do exposto, o presente estudo tem como objetivo analisar aspectos clínicos e epidemiológicos dos tumores cerebrais primários e metastáticos entre os pacientes atendidos no Hospital do Servidor Público Estadual de São Paulo durante os anos de 2010 a 2012.

\section{Métodos}

A presente pesquisa realizou estudo transversal, retrospectivo, do tipo observacional, com análise da incidência de tumores intracranianos primários e metastáticos durante o período de 2010 a 2012 no Hospital do Servidor Público Estadual de São Paulo. Para tanto, foram analisados os dados no setor de registro hospitalar, os prontuários da instituição e registros anatomopatológicos dos pacientes tratados cirurgicamente.

Foram incluídos os dados referentes ao período de janeiro de 2010 a dezembro de 2012, constando: sexo, procedência, idade, localização do tumor, tipo histológico, realização de cirurgia, quimioterapia e/ou radioterapia, recidivas e ocorrência de óbito. Dados incompletos e/ou inconclusivos foram excluídos, assim como tumores da medula espinhal e cauda equina. Foram analisados inicialmente 493 pacientes com o diagnóstico genérico de "Tumores Intracranianos" (CID-10: C 71, D 43, C 71.9, D 32, D 33). Após comparação entre os registros clínicos e resultados de histopatológicos, foram selecionados 369 pacientes, reagrupados segundo tipo histológico, topografia, dados individuais, ocorrência de óbito, recidivas e tipo de tratamento (cirurgia, radioterapia e quimioterapia). Os pacientes foram subdivididos em dois grupos: metástases cerebrais e tumores intracranianos primários. Os dados obtidos foram analisados e reagrupados obedecendo à classificação da OMS para tumores cerebrais. ${ }^{6}$ No grupo das metástases, a localização e o tipo histológico do tumor primário, assim como a quantidade de lesões, também foram avaliados.

As diversas variáveis analisadas foram organizadas em banco de dados tabulado para posterior processamento nos softwares Microsoft Office Excel 2007 e BioEstat 5.0, 
com a confecção de gráficos e tabelas para organização e sistematização dos dados e suas conclusões.

\section{Resultados}

Foram analisados 369 pacientes atendidos no Serviço de Neurocirurgia do Hospital do Servidor Público Estadual de São Paulo, no período de janeiro de 2010 a dezembro de 2012 , sendo 240 do sexo feminino (65\%) e 129 do sexo masculino (35\%), com idade média de 62 anos, sendo a idade mínima de 4 anos e a idade máxima de 97 anos. A idade média nos pacientes do sexo feminino foi de 58,7 anos e nos do sexo masculino, de 65,3 anos. Maior prevalência foi encontrada na faixa etária dos 41 aos 64 anos (191 pacientes, 52\% do total dos casos analisados) (Figura 1). Quanto ao diagnóstico, foram 254 casos de tumores cerebrais primários (69\%) e 115 casos de metástases cerebrais (31\%) (Figuras 2 e 3).

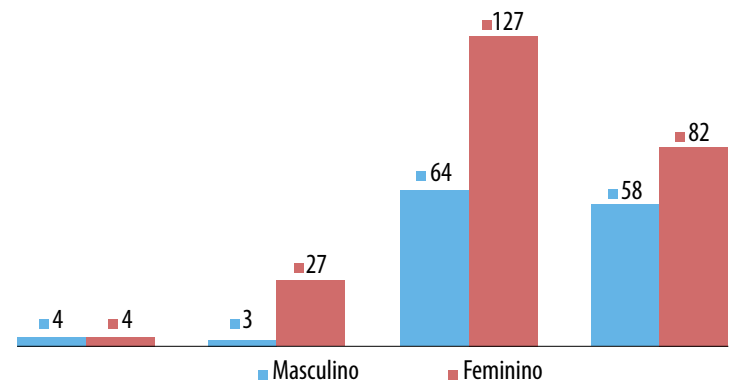

Figura 1 - Distribuição do número de casos de acordo com as faixas etárias.

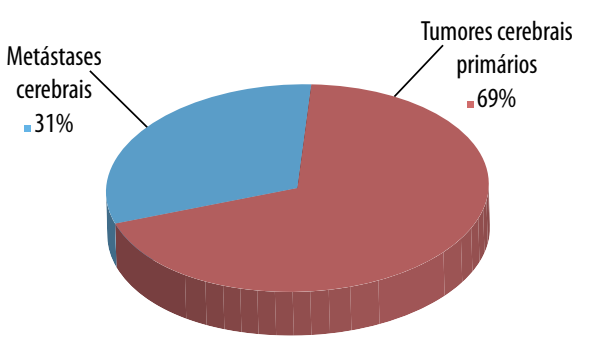

Figura 2 - Comparação entre as incidências de tumores cerebrais primários e metástases cerebrais.

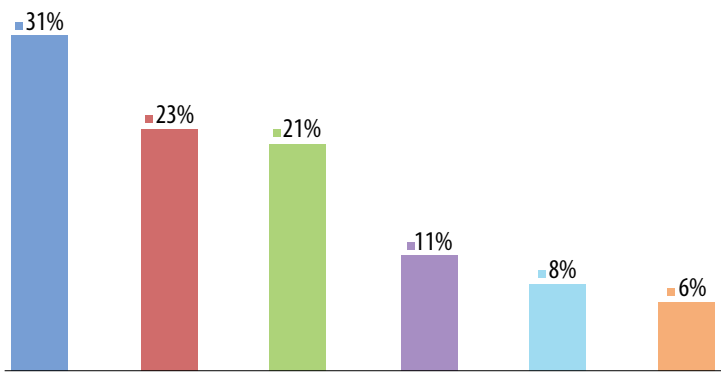

Figura 3 - Comparação entre metástases e tumores intracranianos mais encontrados.
Entre os tumores cerebrais primários, os tumores das meninges foram os mais prevalentes (34\%), seguidos pelos gliomas (30\%), tumores da hipófise (16\%), tumores da bainha neural (10\%), tumores da pineal, neurogliais mistos e embrionários (1\%) (Figura 4). O sexo feminino foi o mais prevalente nos tipos histológicos mais encontrados: meningiomas, glioblastomas, metástases e tumores da hipófise (Figura 5). As meninges foram a localização mais frequente (34\%) (Figura 6). O glioblastoma foi o glioma mais encontrado, correspondendo a $70 \%$ dos casos, seguido pelos astrocitomas benignos (20\%) (Figura 7).

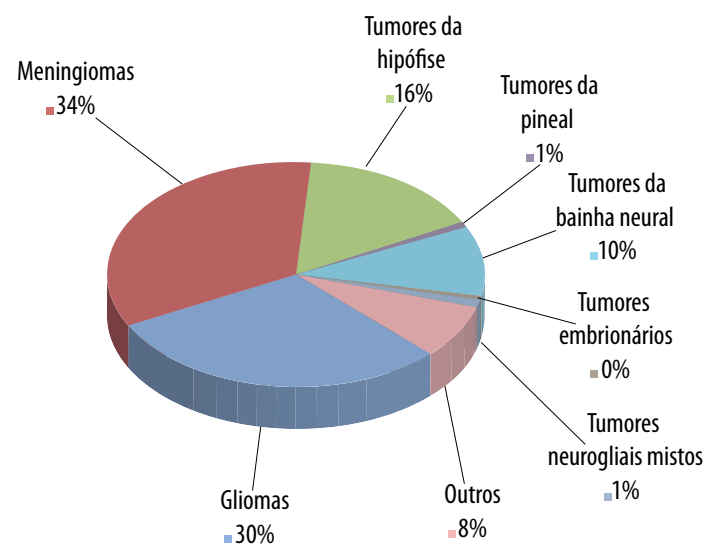

Figura 4 - Tumores intracranianos primários - distribuição de acordo com a histologia.

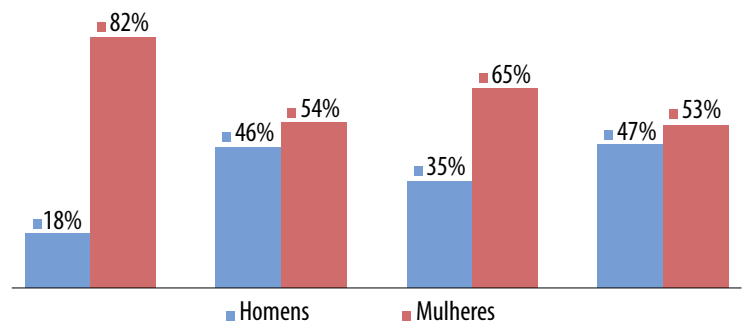

Figura 5 - Comparação entre as prevalências dos diferentes tipos de neoplasias de acordo com o sexo.

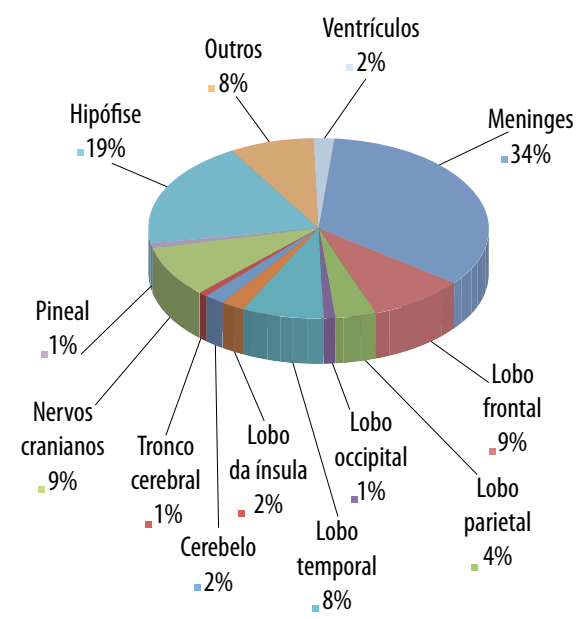

Figura 6 - Localizações mais frequentes de todos os tumores intracranianos primários. 


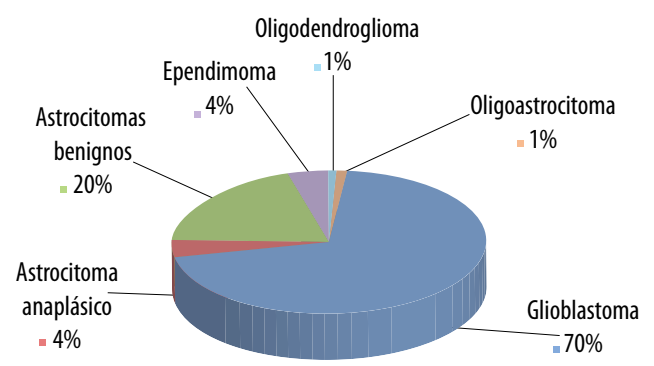

Figura 7 - Distribuição da prevalência entre o grupo dos gliomas. Nota-se predomínio absoluto do glioblastoma (70\%).

No grupo das metástases, as neoplasias de pulmão foram a localização primária mais frequente, com $40 \%$; as neoplasias de mama corresponderam a $24 \%$ dos casos, seguidas pelos cânceres do trato gastrointestinal (6\%) (Figura 8). A maior parte das metástases acometeu o lobo frontal (26\%), seguido pelo cerebelo (25\%), lobo parietal (18\%), lobo temporal (13\%), lobo occipital (4\%) e tronco cerebral (3\%) (Figura 9). Metástases múltiplas ocorreram em $26 \%$ dos casos estudados.

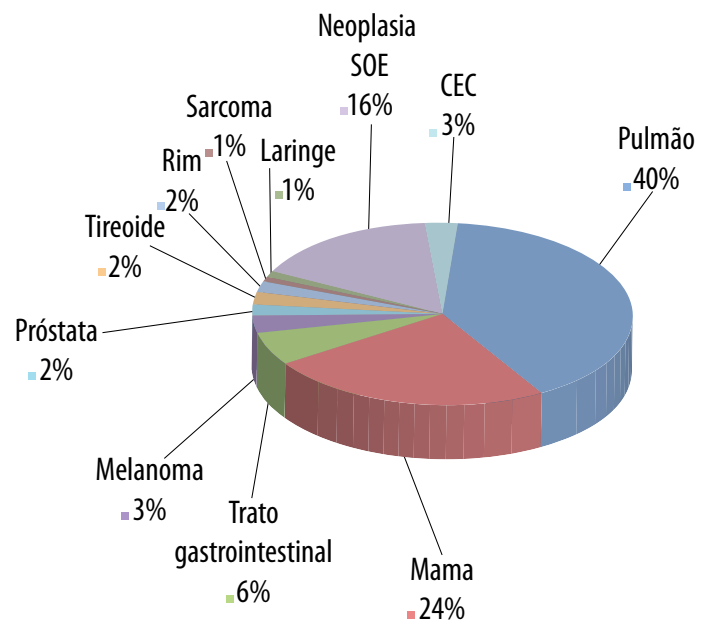

Figura 8 - Localização primária das metástases cerebrais.

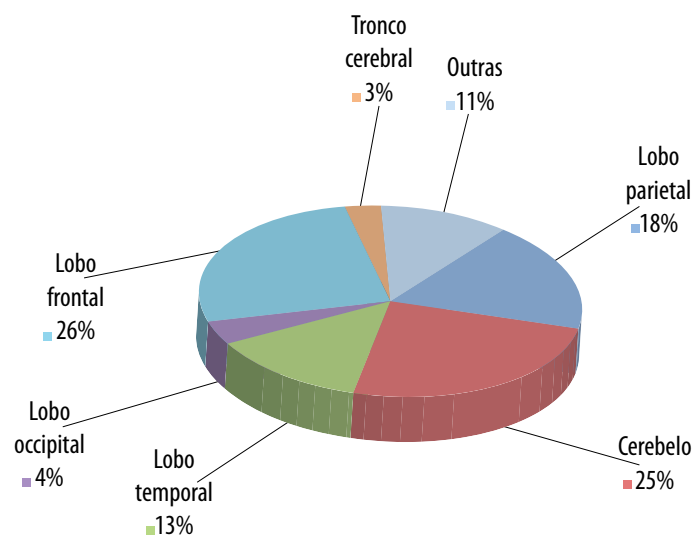

Figura 9 - Metástases - distribuição de acordo com a localização no SNC.

\section{Discussão}

Embora a prevalência de câncer seja o melhor indicador para analisar a sobrevida dessa doença e as potenciais implicações profiláticas necessárias em longo prazo, as incidências dos cânceres, especialmente os que acometem o SNC, não são comumente relatadas. Poucos registros de tumor satisfazem as exigências de dados para uma real estimativa da situação brasileira; com análises regionais satisfatórias, as estatísticas poderiam ser mais realistas. Ainda mais importante, tais estatísticas fornecem a base para o planejamento de saúde, tais como tomadas de decisão na fabricação de medicamentos e de pesquisas, no financiamento para os serviços de saúde e investigação dos fatores de risco.

Cada tipo de tumor localizado no SNC, seja ele primário ou metastático, tem a sua própria biologia, tratamento, prognóstico e suscetibilidade a diferentes fatores de risco. E tumores considerados benignos podem ser letais por causa de sua localização no cérebro, sua capacidade de se infiltrar localmente e sua tendência à malignização, o que torna a classificação de tumores cerebrais uma ciência difícil e cria problemas na descrição epidemiológica dessa patologia. ${ }^{6,11}$

No presente estudo, foi notável a predominância do sexo feminino em todos os principais tipos histológicos, bem como dos indivíduos entre 41 e 64 anos, com média de idade de 62 anos. A predileção pelo sexo feminino analisada corrobora os estudos de Kohler et al. ${ }^{13}$ no entanto discorda das proporções relativamente iguais de tumores cerebrais entre os gêneros comumente relatadas na literatura. ${ }^{14-16} \mathrm{~A}$ maior representatividade dos pacientes maiores de 60 anos também é descrita no relatório da CBTRUS (Central Brain Tumor Registry of the United States) ${ }^{16}$ e de Dobes et al., ${ }^{17}$ apesar das distintas incidências entre os diferentes tipos de tumores analisados neste estudo, fato não comprovado pela presente pesquisa.

Segundo o relatório da CBTRUS ${ }^{16}$, as taxas de incidência para a maioria dos gliomas, schwannomas, tumores de células germinativas e linfomas foram maiores em homens ou equivalentes entre os sexos em todas as faixas etárias, enquanto as mulheres possuíam incidência muito maior de meningiomas e tumores da região selar do que os homens. Neste estudo, o sexo feminino teve maior representatividade em todos os principais tipos histológicos, dado que corrobora estudos mais recentes ${ }^{13,18}$ os quais indicam maior taxa de prevalência de tumores cerebrais entre as mulheres.

Os meningiomas foram os tumores cerebrais primários mais encontrados, seguidos pelos gliomas. Esses dados corroboram os estudos mais recentes como os de Wöhrer et al. ${ }^{19}{ }^{19}$ CBTRUS $^{16}$, Rockhill et al. ${ }^{20} \mathrm{e}$ Barnholtz-Sloan e Kruchko ${ }^{21}$ e discordam de casuísticas clássicas sobre o tema, como a de Wen-Qing et al. ${ }^{22}$ na 
China. Os meningiomas ocorrem principalmente em pacientes entre 40 e 70 anos e são mais comuns no sexo feminino, com taxa de incidência 2,3 vezes maior em mulheres do que em homens (12,42 por $100.000 \mathrm{em}$ mulheres e 5,46 por 100.000 em homens), ${ }^{16}$ aspectos esses ratificados pelo presente estudo. Esses tumores localizam-se preferencialmente na convexidade cerebral, foice, tenda cerebelar, sulcos olfatórios e asa menor do osso esfenoide. A maioria dos meningiomas é considerada benigna (92,8\%), somente $2,2 \%$ são considerados atípicos e $5 \%$ são considerados malignos. ${ }^{18} \mathrm{~A}$ exposição à radiação ionizante, fatores hormonais, polimorfismos genéticos, diabetes, tabagismo, hipertensão, epilepsia e atopia são considerados fatores de risco. ${ }^{18}$

Os gliomas foram o segundo tipo histológico mais encontrado. Analisando separadamente esse grupo, nota-se o predomínio do glioblastoma, dado que corrobora a maior parte da literatura acerca do tema. ${ }^{23-28} \mathrm{Na}$ presente casuística, o glioblastoma foi mais frequente em mulheres, diferentemente de boa parte da literatura, que o considera mais comum em homens. ${ }^{29,30}$ Esse é um tumor cuja frequência aumenta com a idade, sendo raro em crianças. O glioblastoma é o tumor grau IV, maligno, cujas características histopatológicas de alta celularidade, atipias nucleares, infiltração, necrose, proliferação vascular e seu caráter recidivante o tornam de difícil tratamento, com baixas taxas de sobrevida (apenas $2,2 \%$ dos pacientes sobrevivem por mais de três anos após o diagnóstico) e elevadas taxas de recidivas. ${ }^{30} \mathrm{Os}$ gliomas de baixo grau corresponderam a $26 \%$ de todos os gliomas, porém, quando comparados com todos os tumores cerebrais primários, obtiveram discreta representatividade, apenas $8 \%$ do total.

Os tumores cerebrais primários constituíram a maior parte das neoplasias analisadas, em detrimento das metástases para o SNC, o que discorda da maior parte da literatura sobre o tema. ${ }^{31-34}$ Ressalta-se a existência de um viés de seleção, considerando que, apesar de a remoção cirúrgica ser um dos pilares no tratamento das metástases cerebrais, o manejo dessa complicação é individualizado e tem como principais opções, além da cirurgia: corticoterapia, radioterapia, quimioterapia e, cada vez com mais destaque, a radiocirurgia. Desse modo, os casos analisados no presente estudo foram os encaminhados para a neurocirurgia, tratados cirurgicamente e com confirmação histopatológica, o que diminui consideravelmente a casuística. Dados semelhantes foram encontrados no estudo de Correa et al. ${ }^{23}$ que, ao analisar a casuística de um serviço de neurocirurgia de um hospital-escola, também encontrou predomínio dos tumores cerebrais primários em detrimento das metástases. A cirurgia visa restabelecer o quadro funcional dos pacientes, com melhora dos sintomas neurológicos e confirmação diagnóstica, promover paliação imediata e estender a duração do controle no SNC da doença, respeitando os preceitos de acessibilidade e ressecabilidade, porém, apesar de o tratamento cirúrgico seguido de radioterapia ter se mostrado superior em relação à sobrevida e recidiva local, comparativamente com a radioterapia isolada, há tendência crescente de realização da radiocirurgia nas lesões próximas a áreas eloquentes. ${ }^{34}$

Metástases únicas foram maioria em nosso estudo, provavelmente pelo fato de o tratamento cirúrgico ser, principalmente, proposto aos pacientes com metástase cerebral quando há lesão única, em local acessível e controle sistêmico da neoplasia primária; em lesões múltiplas, a sintomática deve ser ressecada e se alguma outra lesão puder ser removida pela mesma via de acesso. ${ }^{8}$ Considerando que a localização da metástase e sua relação com áreas eloquentes do cérebro determinam a manifestação clínica e a orientação terapêutica, o estadiamento da doença primária é um dos determinantes da melhor forma de tratamento das metástases intracranianas; relativamente a pacientes com doença sistêmica controlada ou ausente, o número, tamanho, localização dessas lesões e a condição clínica determinam a melhor forma de tratamento. ${ }^{7,8}$

Os fatores prognósticos envolvidos nesse grupo de pacientes incluem idade, sítio primário, presença de metástases em outros sítios anatômicos, sensibilidade do tumor à terapia, status de Karnofsky e o número e local das lesões no SNC. A ressecção cirúrgica, quando possível, seguida de radioterapia é o tratamento clássico das metástases intracranianas; essa conduta aumenta a sobrevida e, principalmente, o tempo de independência funcional do paciente; além disso, é procedimento indicado em lesões cujo tumor primário é desconhecido e em lesões que produzam hipertensão intracraniana. ${ }^{8}$ A radiocirurgia estereotáxica destaca-se como terapêutica importante nos pacientes com lesões únicas e em áreas eloquentes, e alguns autores a consideram como a melhor opção de tratamento. ${ }^{31}$

Embora qualquer tipo de câncer possa metastatizar para o SNC, a incidência de metástases varia muito com a histologia. Em nosso estudo, o carcinoma de pulmão foi o tipo mais comum de câncer com metástase para o SNC, dado bem aceito pela literatura, cuja incidência varia de $18 \%$ a $65 \%,{ }^{35-39}$ e que também considera que essas metástases ocorrem independentemente do controle da doença primária. As neoplasias de mama foram o segundo tipo histológico mais encontrado, seguidas pelas neoplasias do trato gastrointestinal e pelo melanoma, achados congruentes com a literatura. ${ }^{35,40-42}$ Ressalta-se que pacientes jovens e na fase pré-menopausa estão mais sujeitas a desenvolver metástases cerebrais durante a evolução de um câncer de mama do que pacientes idosas e na fase pós-menopausa, entretanto o presente estudo encontrou predomínio de neoplasias intracranianas nas faixas etárias mais avançadas. ${ }^{42-44}$ 
Tumores de localização primária desconhecida constituíram $16 \%$ da amostra, dados congruentes com a literatura especializada, que considera a metástase cerebral como, muitas vezes, a primeira manifestação de um câncer sistêmico. ${ }^{45,46}$ Apesar da pouca representatividade dos melanomas em nossa casuística, é válido ressaltar que esses tumores apresentam maior propensão para metastatizar para o SNC, incidência essa que varia entre $6 \%$ e $43 \%$ nas séries clínicas e $12 \%$ e $90 \%$ nas séries de autópsias. $^{47-49}$ Quanto à localização, o lobo frontal e o cerebelo foram os locais mais encontrados. Ressalta-se a predileção das metástases pela porção supratentorial (80\% a $85 \%$ dos casos), na transição entre a substância branca e a cinzenta, na região irrigada pelas artérias cerebrais médias. ${ }^{34,50}$ As metástases para o cerebelo possuem um prognóstico pior em comparação com metástases supratentoriais, em virtude da maior propensão de essas lesões causarem hidrocefalia, hérnia, compressão do tronco cerebral e morte. ${ }^{51}$

\section{Conclusão}

Tumores intracranianos primários foram predominantes na casuística. Desses, os meningiomas e os gliomas corresponderam aos principais tipos histológicos. $\mathrm{O}$ glioblastoma foi o glioma mais encontrado. A maioria dos pacientes era do sexo feminino, na faixa etária de 41 a 64 anos de idade, com média de 62 anos de idade. As neoplasias de pulmão, mama e do trato gastrointestinal foram, em ordem decrescente, as localizações primárias mais frequentes das metástases intracranianas; estas tiveram predileção pelo lobo frontal e cerebelo e foram únicas, em sua maioria. Ressaltam-se o considerável aumento da incidência desses tumores com o progredir da idade e a importância dessas neoplasias quanto à morbidade e à mortalidade, assim como para o prognóstico de diversos cânceres.

Há interesse cada vez maior no entendimento da etiologia dos tumores do SNC e estudos epidemiológicos reforçam esse entendimento, porque tais análises descritivas caracterizam a incidência de tumores cerebrais e as taxas de mortalidade com relação ao tipo histológico do tumor e as características demográficas. O intuito é que esse conhecimento resulte em melhores e mais eficazes regimes de tratamento e estratégias individualizadas, de acordo com as características regionais/locais.

\section{Conflito de interesses}

Os autores declaram ausência de quaisquer conflitos de interesses na execução do artigo.

\section{Referências}

1. El-Zein R, Minn AY, Wrensch M, Bondy ML. Epidemiology of brain tumors. Cancer in the nervous system. New York: Oxford University Press; 2002. p. 252-66.

2. Samuels MA. Manual de neurologia: diagnóstico e tratamento. 7a ed. Rio de Janeiro: Revinter; 2007.

3. Ministério da Saúde. Instituto Nacional de Câncer José Alencar Gomes da Silva. Estimativa 2012 - Incidência de câncer no Brasil. Rio de Janeiro: INCA; 2011.

4. Cambruzzi E, Zettler CG, Zettler EW, Jotz GP, Grudzinski M, Pedrini J. Perfil imunoistoquímico de metástases do sistema nervoso central. Rev AMRIGS. 2009;53(4):382-7.

5. Dolecek TA, Propp JM, Stroup NE, Kruchko C. CBTRUS statistical report: primary brain and central nervous system tumors diagnosed in the United States in 2005-2009. Neuro Oncol. 2012;14(Suppl 5):1-49.

6. Louis DN, Ohgaki H, Wiestler OD, Cavenee WK, Burger PC, Jouvet A, et al. The 2007 WHO classification of tumours of the central nervous system. Acta Neuropathol. 2007;114(2):97-109.

7. Gomes JC, Sá A, Pinto RR, Muñoz V, Coelho C, Monsanto F. Tratamento radiocirúrgico de metástases cerebrais: a relação da histologia, localização cerebral e sintomas. Saúde \& Tecnologia. 2012;7(1):28-32.

8. Onishi FJ, Melo JGSP, Melo PMP, Lanzoni OP, Settanni F, Ferraz FAP. Tratamento cirúrgico de metástases intracranianas. Rev Neurociências. 2005;13(1):11-6.

9. Santos AJ, Franco CMR, Borges LRR, Malheiros SMF, Gabbai AA. Metástases cerebrais. Rev Neurociências. 2001;9(1):20-6.

10. Maldaun MVC. Radiocirurgia no tratamento das metástases cerebrais: resposta terapêutica e complicações baseadas na localização das lesões [tese]. São Paulo: Faculdade de Medicina da Universidade de São Paulo; 2006.

11. Porter KR, McCarthy BJ, Freels S, Kim Y, Davis FG. Prevalence estimates for primary brain tumors in the United States by age, gender, behavior, and histology. Neuro Oncol. 2010;12(6):520-7.

12. McDermott MW, Sneed PK. Radiosurgery in metastatic brain cancer. Neurosurgery. 2005;57(Suppl 5):45-53.

13. Kohler BA, Ward E, McCarthy BJ, Schymura MJ, Ries LA, Eheman $C$, et al. Annual report to the nation on the status of cancer, 1975-2007, featuring tumors of the brain and other nervous system. J Natl Cancer Inst. 2011;103(9):714-36.

14. McCarthy BJ, Schellinger KA, Propp JM, Kruchko C, Malmer B. A case for the worldwide collection of primary benign brain tumors. Neuroepidemiology. 2009;33(3):26875.

15. Wrensch M, Minn Y, Chew T, Bondy M, Berger MS. Epidemiology of primary brain tumors: current concepts and review of the literature. Neuro Oncol. 2002;4(4):278-99.

16. Central Brain Tumor Registry of the United States. CBTRUS statistical report: primary brain and central nervous system tumors diagnosed in the United States in 2004-2008. 2012. Disponível em: <http:/ www.cbtrus.org>.

17. Dobes M, Shadbolt B, Khurana VG, Jain S, Smith SF, Smee $\mathrm{R}$, et al. A multicenter study of primary brain tumor incidence in Australia (2000-2008). Neuro Oncol. 2011;13(7):783-90.

18. Claus EB, Bondy ML, Schildkraut JM, Wiemels JL, Wrensch $\mathrm{M}$, Black PM. Epidemiology of intracranial meningioma. Neurosurgery. 2005;57(6):1088-95.

19. Wöhrer A, Waldhör T, Heinzl H, Hackl M, Feichtinger J, Gruber-Mösenbacher U, et al. The Austrian Brain Tumour Registry: a cooperative way to establish a population-based brain tumour registry. J Neurooncol. 2009;95(3):401-11. 
20. Rockhill J, Mrugala M, Chamberlain MC. Intracranial meningiomas: an overview of diagnosis and treatment. Neurosurg Focus. 2007;23(4):E1.

21. Barnholtz-Sloan JS, Kruchko C. Meningiomas: causes and risk factors. Neurosurg Focus. 2007;23(4):E2.

22. Wen-Qing H, Shi-Ju Z, Qing-Sheng T, Jian-Qing H, Yu-Xia L, Qing-Zhong X, et al. Statistical analysis of central nervous system tumors in China. J Neurosurg. 1982;56(4):555-64.

23. Correa C, Soares MS, Cabrera HTN, Figueiredo EG, Teixeira MJ. Gliomas: casuística cirúrgica em um hospital-escola quaternário. Arq Bras Neurocir. 2011;30(1):34-7.

24. Santos R, Frigeri L, Ordovás C, Frigeri M, Bertuol I, Guimarães CVA, et al. Epidemiologia dos tumores do sistema nervoso central, Hospital Nossa Senhora de Pompeia, Serviço de Neurocirurgia, a propósito de 100 casos estudados. Rev Cient AMECS. 2001;10(1):24-32.

25. Schwartzbaum JA, Fisher JL, Aldape KD, Wrensch M. Epidemiology and molecular pathology of glioma. Nat Clin Pract Neurol. 2006;2(9):494-503.

26. Davis FG, Kupelian V, Freels S, McCarthy B, Surawicz T. Prevalence estimates for primary brain tumors in the United States by behavior and major histology groups. Neuro Oncol. 2001;3(3):152-8.

27. Deorah S, Lynch CF, Sibenaller ZA, Ryken TC. Trends in brain cancer incidence and survival in the United States: Surveillance, Epidemiology, and End Results Program, 1973 to 2001. Neurosurg Focus. 2006;20(4):E1.

28. Pereira ELR, Rodrigues DB, Viegas MLC, Hermes Junior MN, Burbano RMR. Epidemiologia dos gliomas no estado do Pará - Parte I (2000-2006). Arq Bras Neurocir. 2012;31(4):200-6.

29. Ohgaki H. Epidemiology of brain tumors. Methods Mol Biol. 2009;472:323-42.

30. Kleihues P, Cavenee WK. Tumors of the central nervous system: pathology and genetics. Lyon: International Agency for Research on Cancer; 1997.

31. Sheehan J, Kondziolka D, Flickinger J, Lunsford LD. Radiosurgery for patients with recurrent small cell lung carcinoma metastatic to the brain: outcomes and prognostic factors. J Neurosurg. 2005;102(Suppl):247-54.

32. Gavrilovic IT, Posner JB. Brain metastases: epidemiology and pathophysiology. J Neurooncol. 2005;75(1):5-14.

33. Schuette $W$. Treatment of brain metastases from lung cancer: chemotherapy. Lung Cancer. 2004;45(Suppl 2):S253-7.

34. Maldaun MVC, Pires de Aguiar PH, Zambelli HJL, Sawaya R. Tratamento cirúrgico das metástases cerebrais. J Bras Neurocirurg. 2006;17(1):14-9.

35. Pan HC, Sheehan J, Stroila M, Steiner M, Steiner L. Gamma knife surgery for brain metastases from lung cancer. J Neurosurg. 2005;102(Suppl):128-33.

36. Kamada K, Mastuo T, Tani M, Izumo T, Suzuki Y, Okimoto $\mathrm{T}$, et al. Effects of stereotactic radiosurgery on metastatic brain tumors of various histopathologies. Neuropathology. 2001;21(4):307-14.

37. Bajard A, Westeel V, Dubiez A, Jacoulet P, Pernet D, Dalphin $\mathrm{JC}$, et al. Multivariate analysis of factors predictive of brain metastases in localized non-small cell lung carcinoma. Lung Cancer. 2004;45(3):317-23.
38. Burt M, Wronski M, Arbit E, Galicich JH. Resection of brain metastases from non-small-cell lung carcinoma. Results of therapy. Memorial Sloan-Kettering Cancer Center Thoracic Surgical Staff. J Thorac Cardiovasc Surg. 1992;103(3):399-410.

39. Schuette $\mathrm{W}$. Treatment of brain metastases from lung cancer: chemotherapy. Lung Cancer. 2004;45(Suppl 2):253-7.

40. Hernandez L, Zamorano L, Sloan A, Fontanesi J, Lo S, Levin $\mathrm{K}$, et al. Gamma knife radiosurgery for renal cell carcinoma brain metastases. J Neurosurg. 2002;97(Suppl 5):489-93.

41. Hoshi S, Jokura H, Nakamura H, Shintaku I, Ohyama C, Satoh M, et al. Gamma-knife radiosurgery for brain metastasis of renal cell carcinoma: results in 42 patients. Int J Urol. 2002;9(11):618-25.

42. Sheehan JP, Sun MH, Kondziolka D, Flickinger J, Lunsford LD. Radiosurgery in patients with renal cell carcinoma metastasis to the brain: long-term outcomes and prognostic factors influencing survival and local tumor control. $J$ Neurosurg. 2003;98(2):342-9.

43. Mystakidou K, Kouloulias V, Tsilika E, Boviatsis E, Kouvaris $\mathrm{J}$, Matsopoulos G, et al. Is early recognition of radiologically silent brain metastasis from breast cancer beneficial? A retrospective study of 22 cases. Breast Cancer. 2004;11(3):276-81.

44. Slimane K, Andre F, Delaloge S, Dunant A, Perez A, Grenier $J$, et al. Risk factors for brain relapse in patients with metastatic breast cancer. Ann Oncol. 2004;15(11):1640-4.

45. Rudà R, Borgognone $M$, Benech $F$, Vasario $E$, Soffietti R. Brain metastases from unknown primary tumour: a prospective study. J Neurol. 2001;248(5):394-8.

46. Drlicek M, Bodenteich A, Urbanits S, Grisold W. Immunohistochemical panel of antibodies in the diagnosis of brain metastases of the unknown primary. Pathol Res Pract. 2004;200(10):727-34.

47. Atallah E, Flaherty L. Treatment of metastatic malignant melanoma. Curr Treat Options Oncol. 2005;6(3):185-93.

48. Daryanani D, Plukker JT, de Jong MA, Haaxma-Reiche $H$, Nap R, Kuiper $H$, et al. Increased incidence of brain metastases in cutaneous head and neck melanoma. Melanoma Res. 2005;15(2):119-24.

49. Sampson JH, Carter JH Jr, Friedman AH, Seigler HF. Demographics, prognosis, and therapy in 702 patients with brain metastases from malignant melanoma. J Neurosurg. 1998;88(1):11-20.

50. Sawaya R, Ligon BL, Bindal AK, Bindal RK, Hess KR. Surgical treatment of metastatic brain tumors. J Neurooncol. 1996;27(3):269-77.

51. Ghods AJ, Munoz L, Byrne R. Surgical treatment of cerebellar metastases. Surg Neurol Int. 2011;2:159.

\section{Endereço para correspondência}

Daniella Brito Rodrigues

Rua Silva Castro, 559, Guamá

66075-104 - Belém, PA, Brasil

Telefone: (91) 8815-7806

E-mail: britodaniella32@gmail.com 